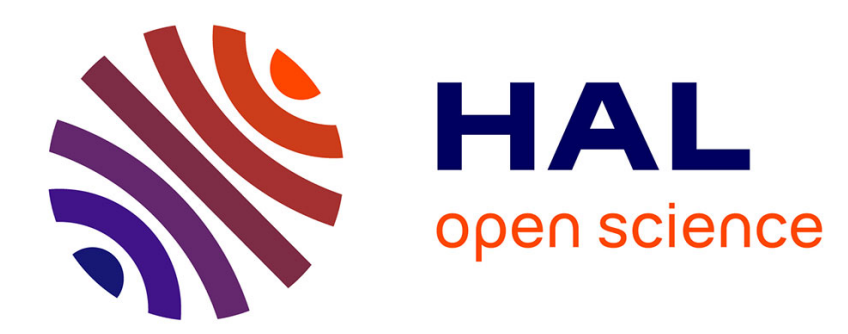

\title{
ESSAIS DE DOSAGE D'ANTIBIOTIQUES DANS LE LAIT
}

P. Dopter

\section{To cite this version:}

P. Dopter. ESSAIS DE DOSAGE D'ANTIBIOTIQUES DANS LE LAIT. Le Lait, 1960, 40 (393_394), pp.151-158. hal-00928265

\section{HAL Id: hal-00928265 \\ https://hal.science/hal-00928265}

Submitted on 1 Jan 1960

HAL is a multi-disciplinary open access archive for the deposit and dissemination of scientific research documents, whether they are published or not. The documents may come from teaching and research institutions in France or abroad, or from public or private research centers.
L'archive ouverte pluridisciplinaire HAL, est destinée au dépôt et à la diffusion de documents scientifiques de niveau recherche, publiés ou non, émanant des établissements d'enseignement et de recherche français ou étrangers, des laboratoires publics ou privés. 


\title{
ESSAIS DE DOSAGE D'ANTIBIOTIQUES DANS LE LAIT
}

\author{
par \\ P. DOPTER
}

Laboratoire des Fermentations de l'Institut Pasteur de Lille

Pour déterminer la quantité d'antibiotique présente dans un lait, on peut avoir recours soit aux méthodes bactériologiques classiques, soit à des méthodes acidimétriques.

Les premières peuvent être employées quelle que soit la concentration de l'antibiotique; c'est ainsi que J. JACQUET et $M^{\text {me }}$ STEeg [1] ont pu déterminer le nombre d'unités de pénicilline, d'auréomycine et de streptomycine seules ou associées dans le lait. Ces méthodes sont précises, mais elles nécessitent un personnel qualifié, et une durée d'incubation qui nuit à la rapidité des résultats.

Les méthodes acidimétriques sont plus rapides; elles sont basées sur la détermination du retard d'acidification du lait ensemencé par un ferment lactique sensible à l'antibiotique recherché. Mais leur emploi est forcément limité par la concentration de cette substance. On sait en effet que chaque antibiotique présente visà-vis de chaque espèce de ferment lactique, d'abord un seuil de toxicité, puis une dose d'inhibition. C'est ainsi que Pien, Lignac et CLAUDE [2] ont indiqué, pour une espèce de Streptococcus lactis, les chiffres suivants :

\section{Seuil de toxicité}

Pénicilline ...........

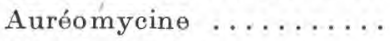

Streptomycine .........

$$
\begin{aligned}
& 0,01 \mathrm{U} . \mathrm{O} \cdot / \mathrm{ml} . \\
& 0,10 \mathrm{\gamma} / \mathrm{ml} . \\
& 0,50 \mathrm{\gamma} / \mathrm{ml} .
\end{aligned}
$$

$$
\begin{gathered}
\text { Dose inhibitrice } \\
0,30 \mathrm{U} . \mathrm{O} \cdot / \mathrm{ml} . \\
0,50 \mathrm{\gamma} / \mathrm{ml} . \\
5,00 \mathrm{\gamma} / \mathrm{ml} .
\end{gathered}
$$

Les déterminations acidimétriques ne seront donc possibles que pour des quantités d'antibiotiques inférieures aux doses inhibitrices. C'est pourquoi un certain nombre d'auteurs préparent d'abord des dilutions décroissantes du lait à tester et, pour éviter de trop nombreux titrages acidimétriques, suivent la marche de l'acidification au moyen d'indicateurs colorés comparativement avec une gamme d'échantillons étalonnés.

Nous avons pu d'autre part montrer [3] que la méthode décrite par Lemoigne, Sanchez et Girard [4] pouvait être étendue à la détection dans le lait des trois antibiotiques (auréomycine, pénicilline, streptomycine) utilisés seuls ou associés en thérapeutique vétérinaire pour lutter contre les mammites. Nous avions signalé, à cette occasion, que nos souches de Streptococcus lactis accoutumées 
à ces antibiotiques acidifiaient moins rapidement et moins fortement le lait que la souche dont elles provenaient.

Ceci nous a conduit à déterminer d'abord le pouvoir acidifiant de nos souches accoutumées, comparativement avec celui de la souche initiale normale, et ensuite à étudier l'influence de doses croissantes d'antibiotiques sur la marche de l'acidification d'un lait ensemencé avec les souches spécifiquement accoutumées.

\section{A. - Pouvoir acidifiant des souches accoutumées.}

La désignation des souches testées est la suivante :

A $5-S$. lactis accoutumé à $5 \gamma$ d'auréomycine par ml.,

P $5-S$. lactis accoutumé à 5 U. O. de pénicilline par ml.,

$\mathrm{S} 5$ - S. lactis accoutumé à $5 \gamma$ de streptomycine par $\mathrm{ml}$.,

$\mathrm{S} \mathrm{N}-\mathrm{S}$. lactis non accoutumé.

Le lait utilisé a été obtenu par dissolution de 100 grammes de lait écrémé non sucré en poudre dans un litre d'eau distillée. Après filtration, le lait a été réparti dans des ballons de 200 millilitres, à raison de 100 millilitres par ballon, et stérilisé par tyndallisation.

Ces ballons ont été ensuite ensemencés au taux de $1 \%$ respectivement avec chacune des souches indiquées, âgées de 24 heures, et mis en incubation à $30^{\circ}$.

Dans chaque ballon, l'acidité Dornic a été déterminée après 5 heures, 8 heures, 12 heures et 24 heures. Les résultats obtenus sont réunis dans le tableau $I$.

\section{TABLEAU I}

POUVOIR ACIDIFIANT COMPARATIF DES SOUGHES AGCOUTUMÉES ET DE LA SOUGHE INITIALE

Souches
Acidité (en degrés Dornic) après :

0 h. $\quad 5 \mathrm{~h} . \quad 8 \mathrm{~h} . \quad 12 \mathrm{~h} . \quad 24 \mathrm{~h}$.

$\begin{array}{llllll}\text { A } 5 \ldots \ldots \ldots \ldots \ldots & 15 & 21 & 32 & 58 & 77 \\ \text { P } 5 \ldots \ldots \ldots \ldots & 15 & 21 & 29 & 59 & 73 \\ \text { S } 5 \ldots \ldots \ldots \ldots & 15 & 26 & 56 & 72 & 81 \\ \text { S N } \ldots \ldots \ldots \ldots & 15 & 30 & 65 & 73 & 82\end{array}$

De l'examen de ces chiffres, il ressort que :

- $1^{0}$ Les souches accoutumées ont acidifié moins rapidement et moins fortement le lait que la souche normale;

$2^{\circ}$ Les différences (en degrés Dornic) ont varié suivant les souches et la durée d'incubation; elles ont été de : 


\begin{tabular}{|c|c|c|}
\hline & A 5 & P 5 \\
\hline Après 5 heures ...... & 9 & 9 \\
\hline Après 8 heures $\ldots \ldots$ & 33 & 36 \\
\hline Après 12 heures ..... & 15 & 14 \\
\hline Après 24 heures . . . . . & 5 & 9 \\
\hline
\end{tabular}

Elles ont été importantes pour A 5 et P 5, surtout après 8 heures d'incubation, mais faibles pour $\mathbf{S} 5$.

\section{B. - Influence d'une addition d'antibiotique sur le pouvoir acidifiant des souches accoutumées.}

Les essais ont été conduits suivant le même mode opératoire que précédemment; mais, pour chacune des trois séries, nous avons ajouté au lait, avant ensemencement, des quantités croissantes d'une solution fraîche de l'antibiotique à étudier, sans dépasser toutefois la dose d'accoutumance. Les contrôles acidimétriques ont été effectués comme précédemment après les mêmes temps d'incubation à $30^{\circ}$. Nous avons consigné dans les tableaux II, III et IV, les résultats moyens des essais répétés trois fois chacun.

TABLEAU II

INFLUENGE DE L'AUREOMYGINE

SUR LE POUVOIR AGIDIFIANT DE LA SOUCHE ACGOUTUMÉE

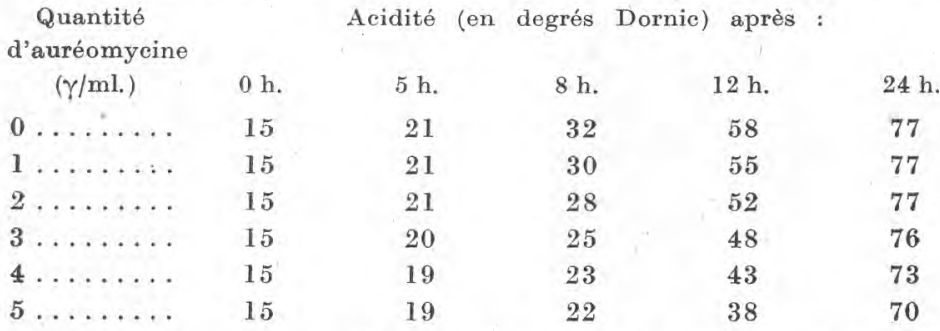

Les différences partielles d'acidité ont été minimes après 5 heures et peu importantes après 24 heures d'incubation. Elles ont été en moyenne de $2^{\circ}$ Dornic par unité d'auréomycine après 8 heures, et de $4^{\circ}$ Dornic après 12 heures.

TABLEAU III

INFLUENCE DE LA PÉNICHLLINE

SUR LE POUVOIR ACIDIFIANT DE LA SOUGHE ACCOUTUMÉE

Quantité de pénicilline

(U. O./ml.) $0 \mathrm{~h}$.

$0 \ldots \ldots \ldots$

$1 \ldots \ldots \ldots$

$2 \ldots \ldots \ldots \quad 15$

$3 \ldots \ldots 15$

$4 \ldots \ldots \ldots \quad 15$

$5 \ldots \ldots \ldots 15$

Acidité (en degrés Dornic) après :

$\begin{array}{crrr}5 \mathrm{~h} . & 8 \mathrm{~h} . & 12 \mathrm{~h} . & 24 \mathrm{~h} . \\ 21 & 29 & 59 & 73 \\ 21 & 28 & 57 & 72 \\ 20 & 28 & 55 & 72 \\ 20 & 27 & 52 & 70 \\ 19 & 27 & 50 & 70 \\ 18 & 26 & 48 & 68\end{array}$


Après 5 heures, 8 heures, et 24 heures, les différences partielles n'ont pas été importantes ; elles l'ont été davantage (en moyenne de $2^{\circ}$ Dornic par unité de pénicilline), après 12 heures.

TABLEAU IV

INFLUENGE DE LA STREPTOMYGINE

SUR LE POUVOIR AGIDIFIANT DE LA SOUCHE ACCOUTUMÉE

Quantité de

streptomycine

$(\gamma / \mathrm{ml}) \quad .0 \mathrm{~h}$

$0 \ldots \ldots \ldots$

$1 \ldots \ldots \ldots$

$2 \ldots \ldots \ldots$

$3 \ldots \ldots \ldots$

$4 \ldots \ldots \ldots$

$5 \ldots \ldots \ldots$

Acidité (en degrés Dornic) après :

$\begin{array}{cccc}5 \mathrm{~h} . & 8 \mathrm{~h} . & 12 \mathrm{~h} . & 24 \mathrm{~h} . \\ 26 & 56 & 72 & 81 \\ 26 & 55 & 72 & 81 \\ 26 & 56 & 71 & 81 \\ 26 & 55 & 72 & 80 \\ 25 & 55 & 70 & 81 \\ 26 & 54 & 71 & 80\end{array}$

On voit que, dans le cas de la streptomycine, les différences partielles ont été négligeables, ce qui n'est pas surprenant quand on connaît la faiblesse d'action de cet antibiotique sur le $S$. lactis, faiblesse qui se traduit par la facilité avec laquelle celui-ci s'y accoutume.

\section{C. - Application.}

La constatation de cette diminution régulière d'acidification après un temps d'incubation de 8 heures ou 12 heures, suivant les cas, nous a conduit à utiliser la mesure de cette diminution pour déterminer la quantité d'antibiotique présente dans le lait.

Nous avons tout d'abord vérifié que la marche de l'acidification d'un lait écrémé et d'un lait entier du commerce, ensemencés avec nos souches accoutumées était identique à celle que nous venions d'étudier avec du lait écrémé reconstitué. Les valeurs absolues des résultats ont quelque peu varié suivant l'acidité initiale de ces laits ; mais les différences partielles n'ont pas changé.

Puis nous avons tenté d'augmenter l'importance de ces différences partielles :

$1^{\circ}$ En faisant varier le taux d'ensemencement: en augmentant celui-ci jusqu'à 4\%, l'acidification du lait a été accélérée, mais les différences partielles ont été diminuées. En le diminuant à $0,5 \%$, celles-ci ont un peu augmenté, mais la durée de l'acidification a dépassé les limites compatibles avec une obtention suffisamment rapide des résultats. Les plus grands écarts constatés correspondaient à un taux de $1 \%$;

$2^{\circ}$ En faisant varier la température d'incubation de $25^{\circ} \grave{a} 35^{\circ}$ : nous avons observé des variations identiques aux précédentes, et avons conservé la température de $30^{\circ}$. 
Ces conditions ayant été établies, nous avons délaissé le dosage de la streptomycine au moyen de la souche accoutumée correspondante : le retard d'acidification n'était en effet pas suffisant pour être interprétable; mais nous avons pu doser l'auréomycine et la pénicilline dans des laits entiers ou écrémés en utilisant la technique suivante :

$1^{0}$ On détermine d'abord l'acidité Dornic initiale du lait à tester ;

$2^{\circ}$ On introduit 50 millilitres de ce lait dans un ballon stérile de 100 millilitres et on maintient celui-ci pendant 10 minutes dans un bain-marie chauffé à $90^{\circ}$;

$3^{\circ}$ Après refroidissement à $30^{\circ}$ environ, on ensemence ce ballon avec $0,5 \mathrm{ml}$. exactement mesuré, d'une culture de 24 heures $\mathrm{du}$ $S$. lactis accoutumé à l'antibiotique à doser. Après une soigneuse homogénéisation, on place le ballon en incubation à $30^{\circ}$ pendant 12 heures;

$4^{\circ}$ Après ce temps, on détermine l'acidité Dornic de la culture, et on en déduit l'augmentation $d$ de cette acidité par rapport à l'acidité initiale.

On prépare en même temps et dans les mêmes conditions, une culture de lait de même nature, mais exempte de substance inhibitrice et on déterminé sur celle-ci, après un même temps d'incubation, la valeur $D$ de l'augmentation de son acidité.

Comme on connaît d'autre part, la diminution moyenne $\Delta$ de degrés Dornic par unité d'antibiotique, la concentration $\mathrm{C}$ de celui-ci exprimée en unité par millilitre, sera :

$$
\mathrm{C}=\frac{D-d}{\Delta}
$$

Il est évidemment essentiel de connaître la valeur de $\Delta$ correspondant à la souche acclimatée. Dans les essais que nous avons exposés, elle était de 4 pour la souche A 5 , et de 2 pour la souche P 5. Si on opérait avec une souche acclimatée inconnue, il faudrait déterminer cette valeur en mesurant, comme nous l'avons indiqué précédemment, les différentes acidités correspondant aux quantités croissantes d'antibiotique ajoutées au lait.

\section{Discussion.}

La précision des dosages est forcément limitée par celle de la détermination d'une acidité Dornic, c'est-à-dire que les chiffres obtenus sont exacts à $0^{\circ} 5$ près pour des laits non caillés, et à $1^{\circ}$ près pour ceux qui le sont. Encore faut-il prendre les précautions suivantes pour avoir des résultats comparables : 
10 Pratiquer les ensemencements avec des cultures de même âge ayant été ensemencées rigoureusement au même taux ;

$2^{\mathrm{e}}$ Avant de procéder au tirage acidimétrique, homogènéiser soigneusement le contenu du ballon, surtout s'il est coagulé.

C'est ainsi que, par cette méthode simple, nous arons pu retrouver dans différents laits les concentrations de 1 à 5 unités d'auréomycine ou de pénicilline que nous leur avions ajoutées. Bien que moins précise que la méthode bactériologique en boîtes de Petri, elle est plus rapide, puisque les résultats peuvent être connus après 12 heures environ.

Pour des teneurs en antibiotiques supérieures au taux d'accoutumance de la souche correspondante, la méthode décrite ne pourra évidemment pas convenir, car l'inhibition serait complète. Bien que les teneurs dépassant 5 unités par millilitre soient rares, il sera bon, par mesure de prudence, de faire simultanément un autre dosage sur le même lait, après l'avoir dilué de moitié avee un lait sain.

Si les teneurs sont inférieures à 1 unité par millilitre, la méthode décrite n'est plus assez sensible : il faut en effet, une différence minima de $2^{\circ}$ Dornic avec l'acidité initiale pour pouvoir déceler 1 unité d'antibiotique par millilitre. On devra alors recourir à la méthode bactériologique de JACQUET dont la précision est d'autant plus grande que les concentrations sont plus faibles.

Il existe aussi la méthode de dosage préconisée par PIEN, Lignac et Cladde [1] qui est une extension de leur technique de détection qualitative au moyen de souches accoutumées. Pour en faire une méthode quantitative, ils ensemencent le lait à tester avec une gamme de souches accoutumées à différentes concentrations connues d'antibiotique, et notent le taux d'accoutumance de la première d'entre elles qui est inhibée après incubation. Au dire même des auteurs, cette détermination n'est qu'approximative. Nous le eroyons d'autant plus volontiers que, en contrôlant nos essais plusieurs mois après les avoir mis au point, nous avons constaté que nos souches accoutumées, malgré la régularité des repiquages effectués dans des conditions toujours identiques, acidifiaient un peu plus fortement les laits (entiers ou écrémés) renfermant les antibiotiques que nous leur avions ajoutés, comme auparavant, à doses croissantes. Il en résultait une diminution sensible des écarts que nous avions constatés.

C'est ainsi que, sans rapporter ici les chiffres détaillés que nous avons trouvés, les écarts différentiels moyens n'étaient plus que de $1^{\circ}$ Dornic pour l'auréomycine, et inférieurs à $1^{\circ}$ Dornic pour la pénicilline, c'est-à-dire absolument ininterprétables.

Comme, d'autre part, le pouvoir acidifiant de ces souches dans 
un lait exempt d'antibiotique n'avait pas augmenté, nous avons pensé que leur taux d'accoutumance s'était accru et que, en dessous des concentrations auxquelles nous les avions acclimatées, ces souches étaient devenues de moins en moins sensibles à la présence des deux antibiotiques étudiés. Nous avons pu vérifier cette hypothèse en contrôlant d'abord le taux d'accoutumance de chacune d'elles, et avons constaté que, après deux années d'entretien régulier, ce taux avait augmenté de $1 \gamma$ par millilitre pour la souche A 5, et de 2 U. O. par millilitre pour la souche P 5. Nous avons alors ramené les nouveaux taux constatés à leurs valeurs primitives par une série de repiquages sur lait dépourvu d'antibiotiques. Nous avons ensuite refait nos essais de dosages avec ces nouvelles souches : nos résultats ont alors confirmé ceux que nous avions tout d'abord obtenus.

Il ressort de tout ceci que, pour obtenir des résultats valables en appliquant notre technique de dosage, il est indispensable de vérifier régulièrement la constance des retards d'acidification produits par les souches utilisées en présence de quantités croissantes des antibiotiques correspondants.

Nos souches accoutumées ne se sont donc pas comportées comme celles de Pien, Lignac et Claude qui, "après plusieurs mois ou "plusieurs années d'entraînement, sont capables de produire une "fermentation parfaitement normale (rapidité, acidité...) dans des "laits renfermant une ou plusieurs fois la concentration inhibi" trice. »

De plus, les variations d'accoutumance qui se sont manifestées après un entretien prolongé montrent qu'elles n'auraient pas pu être utilisées valablement dans la méthode indiquée par PIEN et ses collaborateurs pour déterminer une concentration, même approximative, des antibiotiques dans le lait.

\section{E. - Conclusion.}

Nous avons pu observer en premier lieu que les souches de $S$. lactis que nous avions accoutumées à l'auréomycine, à la pénicilline et à la streptomycine pour la détection de ces antibiotiques dans le lait n'acidifiaient pas celui-ci dans les mêmes conditions que que la souche initiale non acclimatée à ces substances.

En second lieu, les laits renfermant des doses croissantes de ces antibiotiques et ensemencés avec ces mêmes souches, présentaient un retard d'acidification proportionnel aux quantités ajoutées dans les limites de l'accoutumance obtenue. Ce retard, suffisamment important pour pouvoir être chiffré dans le cas de l'auréomycine et de la pénicilline, n'était pas assez significatif dans celui de la streptomycine. 
De ces observations nous avons tiré une méthode simple de dosage de ces deux antibiotiques. Mais la constance des résultats a été altérée par une lente augmentation du taux d'accoutumance de ces souches après un entretien prolongé. Cette constance a pu être rétablie en le ramenant à sa valeur initiale.

La méthode décrite permet de connaître en 12 heures, à 0,5 unité près, la quantité d'auréomycine et de pénicilline existant dans un lait entier ou écrémé, jusqu'à une concentration de 5 unités par millilitre, sans avoir recours aux nombreuses dilutions qui sont nécessitées par l'application de méthodes acidimétriques, à vrai dire expéditives, décrites par certains auteurs pour le dosage de concentrations inférieures à l'unité par millilitre.

Enfin il résulte de nos essais que, pour être valables, les déterminations doivent être faites dans des conditions strictement identiques d'ensemencement et de titrage, en n'utilisant que des souches dont le taux d'accoutumance et le pouvoir acidifiant en présence de quantités croissantes d'antibiotiques ont été de temps en temps soigneusement vérifiés.

\section{Summary}

The method described permits knowledge in twelve hours of the quantity of aureomyein and penicillin contained in whole or skim milk, to the exactitude of 0,5 . It can be used on a concentration up to 5 units per $\mathrm{ml}$, without necessitating recourse to numerous dilutions which are used in acidimetric methods, which may be more rapid, and which are described by certain authors for the determination of concentrations lower than one unit per $\mathrm{ml}$.

In order to be valid, the determinations should be done under strictly identical conditions of insemination and titration, using only species whose acclimatization eapacities and acidifying powers have been carefully verified and tested in the presence of increasing quantities of antibiotics.

\section{BIBLIOGRAPHIE}

[1] J, Jacquet et Mme Steeg. Méthode microbiologique de diagnose et de titrage des antibiotiques dans le lait et les produits laitiers. Ann. Fals. Fraudes, 1953, 46, 5-14.

[2] J. Pien, J. Lignac, P. Cla de Détection biologique des antiseptiques et des antibiotiques dans le lait. Le Lait, 1953, 33, 369-382.

[3] P. Dopter. La recherche d'antibiotiques seuls ou associés dans le lait. C. R. Ac. Agric., 1956, 42, 519-522.

[4] M. Lemoigne, G. Sanchez, H. Girard. Caractérisation de la pénieilline et de la streptomycine dans le lait. C. R. Ac. Agric., 1952, 39, 608. 